\title{
Warum der Euro Europa spaltet statt es zu einigen ${ }^{1}$
}

\section{Wolfgang Streeck}

Zu den besonders ausführlich behandelten Themen im zweiten Kapitel von Max Webers monumentalem Werk Wirtschaft und Gesellschaft, überschrieben "Soziologische Grundkategorien des Wirtschaftens" (1956 [1920]), gehört das Geld. Für den Soziologen Weber wird Geld zu Geld kraft einer „Verbandsordnung“ (ibid., 54), auch „Geldordnung“ (ibid., 125) oder "Geldverfassung" (ibid., 145), die unter modernen Bedingungen, so Weber im Anschluss an Knapps Staatliche Theorie des Geldes (1905), nur eine von einem Staat monopolisierte sein könne (ibid., 125). Geld ist eine in einem Herrschaftsverband - ein weiterer zentraler Weberscher Begriff - ein- und durchgesetzte politisch-ökonomische Institution, die wie alle Institutionen bestimmte Interessen privilegiert und andere benachteiligt. Dies macht es zum Gegenstand gesellschaftlichen „Kampfes“ bzw., als wirtschaftliche Institution, zu einer Ressource in dem, was Weber als „Marktkampf“ bezeichnet. „Geld“, so Weber (ibid., 77)

„ist keine harmlose ,Anweisung auf unbestimmte Nutzleistungen', welche man ohne grundsätzliche Ausschaltung des durch Kampf von Menschen mit Menschen geprägten Charakters der Preise beliebig umgestalten könnte, sondern primär: Kampfmittel und Kampfpreis, Rechnungsmittel aber nur in der Form des quantitativen Schätzungsausdrucks von Interessenkampfchancen."

Webers soziologisch-politischer Geldbegriff unterscheidet sich grundlegend von der Geldtheorie der liberalen Tradition der Wirtschaftswissenschaft. ${ }^{2}$ Deren Gründungsdokument sind die Kapitel IV und V von Adam Smiths Wealth of Nations (1993 [1776]), wo die Entstehung des Geldes als Herausbildung eines immer allgemeiner einsetzbaren Tauschmediums zum Zweck einer letztlich unbegrenzten Erweiterung von Handelsbeziehungen in fort-

\footnotetext{
${ }^{1}$ Distinguished Lecture in the Social Sciences, Wissenschaftszentrum Berlin, 21. April 2015.

${ }^{2}$ Wesentliche Anregungen zu den folgenden Überlegungen verdanke ich Geoffrey Inghams Buch The Nature of Money (2004).
} 
geschrittenen, d.h. arbeitsteilig organisierten Gesellschaften erklärt wird. Geld dient dazu, direkten Tausch durch Zwischenschaltung einer universell verfügbaren, leicht transportierbaren, unendlich teil- und haltbaren Zwischenware in indirekten Tausch zu überführen (bei Marx bekanntlich: erweiterte Reproduktion, $W \rightarrow G \rightarrow W)$. Geldordnungen wachsen Smith zufolge von unten, aus dem Wunsch der Marktteilnehmer nach Ausbau und Vereinfachung ihrer Handelsbeziehungen; sie unterscheiden sich nach den in ihnen anfallenden Transaktionskosten, und werden effizienter, indem sie diese immer weiter senken. Geld ist für Smith ein möglichst zweckmäßig zu gestaltendes, neutrales Symbol für den Wert von Tauschgegenständen, auch wenn es selbst einen objektiven Wert hat (Kapitel V), der sich arbeitswerttheoretisch aus seinen Produktionskosten ergeben soll. Der Staat kommt bei Smith nur insoweit ins Spiel, als er von den Marktteilnehmern zur Steigerung der Effizienz des Geldes gewissermaßen hinzugebeten werden kann, um dieses durch „Abstempelung“ vertrauenswürdiger zu machen. Anders als bei Weber, wo Geldordnungen auch nach ihrer Affinität zu gegenläufigen Verteilungsinteressen unterschieden werden können, ist das einzige Interesse, dem Geld bei Smith mehr oder weniger gut dienen kann, das allgemeine an einem reibungslosen Funktionieren einer möglichst weitreichenden Marktwirtschaft.

Erstaunlicherweise ist es nicht Weber, dem die soziologische Tradition gefolgt ist, sondern Smith. Das Aussterben der Historischen Schule der Nationalökonomie und die Abtretung der Wirtschaft als Forschungsgegenstand durch den Strukturfunktionalismus an eine zunehmend neoklassisch gesäuberte Wirtschaftswissenschaft ${ }^{3}$ erlaubten es der sich im posthistoire der Nachkriegsjahrzehnte etablierenden Soziologie, auf eine eigene Geldtheorie zu verzichten und Geld um des lieben Friedens willen, wenn überhaupt, im Smithschen Sinne als interessenneutrales Kommunikationsmedium statt als machtdurchschossene soziale In-

\footnotetext{
${ }^{3}$ Namentlich durch Talcott Parsons in der von ihm mit dem wirtschaftswissenschaftlichen Fachbereich der Harvard-Universität ausgehandelten Zuständigkeitsverteilung.
} 
stitution - als numéraire statt als soziale Beziehung (Ingham 2004) - aufzufassen. Damit riss

auch in der Soziologie, ebenso wie in der Wirtschaftstheorie, die Erinnerung an die heftigen Auseinandersetzungen der Zwischenkriegszeit über die Natur des Geldes und die politischen Auswirkungen von Geldordnungen ab, wie sie noch im Mittelpunkt gerade auch der Keynesschen Theorie gestanden hatten - siehe die führend von Keynes vorangetriebenen Diskussionen über die sozialen und politischen Implikationen des Goldstandards oder über das Vollgeld-Modell von Irving Fisher. Von paradigmatischer Bedeutung war hier das Buch von Parsons und Smelser, Economy and Society, mit dem Untertitel A Study in the Integration of Economic and Social Theory (1984 [1956]), in dem Geld als Repräsentation von Kaufkraft als der Fähigkeit erscheint, den Austausch von Gütern zu kontrollieren. Als speziell soziale Funktion kommt hinzu, dass es darüber hinaus Prestige verleiht und so zwischen besonderen und allgemeinen Symbolisierungen vermittelt (ibid., 71). Historisch entwickelt sich Geld wie bei Smith im Kontext der Ausweitung der Arbeitsteilung, für die es einer abstrakten Darstellung wirtschaftlichen Werts bedarf, damit erweiterter Austausch möglich wird (ibid., 140 f.). Geld erscheint dabei als „kulturelles Objekt“, zusammen mit Kreditinstrumenten und Schuldscheinen, die „Rechte auf oder Ansprüche an Objekte von ökonomischem Wert repräsentieren“ (ibid., 106) - also mit Weber (s. o.) als „harmlose Anweisung auf unbestimmte Nutzleistungen". ${ }^{4}$

\footnotetext{
${ }^{4}$ Siehe auch Parsons' Aufsatz über „evolutionäre Universalien“ (1964), in dem „Markt und Geld" als eine von vier grundlegenden historischen Errungenschaften moderner Gesellschaften erscheinen, neben bürokratischer Organisation, einem universalistischen Rechtssystem und demokratischen Assoziationsformen. Evolutionäre Universalien sind für Parsons Strukturelemente sozialer Systeme, ohne die deren weitere Entwicklung blockiert wäre. Geld und Markt als zusammenhängender institutioneller Komplex leisten in den Gesellschaften, in denen sie sich herausgebildet haben, „einen fundamentalen Beitrag zu [deren] Anpassungsfähigkeit“ (ibid., 350), weil sie die Herauslösung von Ressourcen aus askriptiven Bindungen und ihre Umwidmung zu neuen Zwecken erleichtern. Dabei ist Geld als „symbolisches Medium“, das den „wirtschaftlichen Nutzen“ der konkreten Güter, gegen die es eintauschbar ist, in abstrakter, gegenüber den Ansprüchen anderer Ordnungen "neutraler" Form „repräsentiert“, unentbehrlich. Geld kann in verschiedenen Gesellschaften unterschiedlich entwickelt sein, wobei seine Funktionen teilweise auch von bürokratischen Organisationen über-
} 
Dass Geld aber viel mehr ist als das, dafür hätten Parsons und die amerikanische Soziologie nicht nur in den nach 1945 irgendwie zu einer Ausnahmezeit erklärten Zwischenkriegsjahren, sondern auch in der weiter zurückliegenden Geschichte ihres Landes reichlich Belege finden können. Deren Entdeckung musste jedoch auf die Neuentstehung einer historisch-institutionalistischen politischen Ökonomie in den 1990er Jahren in Gestalt der „neuen Wirtschaftssoziologie" und die von ihr zu vollziehende Rehabilitierung der Weberschen Perspektive auf Geld als „Kampfmittel und Kampfpreis“ im „Marktkampf“ warten. Einen nach wie vor wichtigen Beitrag hierzu lieferte etwa die 1996 veröffentlichte Untersuchung von Carruthers und Babb über die innenpolitischen Konflikte über eine neue Geldordnung in den USA nach dem Bürgerkrieg (1996). Die Autoren zeigen, im Anschluss an eine von Jack Knight (1992) vorgeschlagene analytische Unterscheidung, dass Geldordnungen, wie Institutionen im allgemeinen, nicht nur als Instrumente einer „Koordinierung zu kollektivem Nutzen“ aufgefasst werden dürfen (,the coordination-for-collective-benefits conception of social institutions"), also nicht allein danach zu beurteilen sind, ob sie eine intersubjektiv kommunizierbaren Symbolisierung von Werten und Wertansprüchen zur Verfügung stellen. Ebenso angemessen und in der Tat gefordert, so Carruthers und Babb, ist die von Knight als solche bezeichnete konflikttheoretische, man könnte auch sagen: politische Perspektive, in der eine Geldordnung als Resultat von Auseinandersetzungen zwischen Akteuren mit unterschiedlichen Interessen erscheint, in welcher Eigenschaft sie mehr oder weniger asymmetrische konkreten Geldordnung die Funktion erfüllen, „die operativen Einheiten der Gesellschaft, einschließlich natürlich ihrer Regierung, mit einem Reservoir von verfügbaren Ressourcen auszustatten, die zu beliebigen Zwecken verwendet und, innerhalb von Grenzen, von Verwendung zu Verwendung verschoben werden können“ (ibid., 350). 
Verteilungseffekte und miteinander in Konflikt liegende Interessen erzeugt, die in der sozialen Realität oft wichtiger sind als ihre Effizienz (1558 f.). ${ }^{5}$

Konkret geht es bei Carruthers und Babb um die Rekonstruktion der wirtschaftlichen, politischen und ideologischen Konfliktlinien in den Auseinandersetzungen in den USA im letzten Drittel des 19. Jahrhunderts um das künftige Geldregime des Landes und die Natur des Geldes im allgemeinen. Dabei verliefen die Fronten ziemlich genau zwischen einer Smithschen und einer Weberschen Konzeption von Geld, wobei die erstere - bei der die Verlässlichkeit des Geldes als Medium symbolischer Kommunikation zum Zweck effizienter wirtschaftlicher Koordinierung und sozialer Integration im Vordergrund stand - mit einer Präferenz für eine Rückkehr zum Goldstandard und einer naturalistischen Werttheorie liiert war, während die letztere, ausgehend von einer bemerkenswert entwickelten politischsozialkonstruktivistischen Theorie des Geldwerts, für frei geschöpftes Papiergeld eintrat. Wie zu erwarten, betonten die Befürworter von Gold das Allgemeininteresse an einer Vertrauen schaffenden Wertsymbolisierung, während die Anhänger der „Greenbacks“ - der gedruckten Dollarscheine - auf die unterschiedlichen Verteilungswirkungen der beiden Geldkonzepte und die von ihnen jeweils bedienten materiellen Interessen verwiesen. In der Tat waren die konkurrierenden Vorstellungen der Anhänger von Gold und Papier über eine neue Geldordnung fest in distinkten Wirtschafts- und Lebensweisen verankert: Während die Befürworter des Goldstandards dem an der Ostküste beheimateten Milieu des alten Geldes angehörten, dessen Interesse vor allem auf Stabilität gerichtet war, hatte die Papiergeldfraktion ihren Sitz

\footnotetext{
${ }^{5}$ Geldordnungen lassen sich in diesem Sinne analog zu politischen Systemen auffassen, in deren Struktur typischerweise eine Tendenz zur Verzerrung von Entscheidungen zugunsten privilegierter Interessen eingebaut ist - eine Eigendynamik, die E. E. Schattschneider in Bezug auf die pluralistische Demokratie der Vereinigten Staaten als „mobilization of bias" bezeichnet hat: „The flaw in the pluralist heaven", so Schattschneider, ",is that the heavenly choir sings with a strong upper-class accent" (1960, 34 f.). Den Hinweis auf Schattschneider verdanke ich einem kürzlichen Aufsatz von Hacker und Pierson (2014). Ingham (2004) beschreibt Geld als „soziale Beziehung“, deren konkrete Gestalt durch die jeweils unterliegende Geldordnung konditioniert wird.
} 
im Süden und Westen und war an laufender Entwertung aufgehäufter Schulden bzw. raschem Wirtschaftswachstum durch freien Zugang zu möglichst unbegrenztem Kredit interessiert. Auf diese Weise, so Carruthers und Babb, verschlangen sich konfligierende Interessen an unterschiedlichen Wachstumspfaden für die sich entfaltende kapitalistische Ökonomie der Vereinigten Staaten mit in Widerstreit liegenden Machtansprüchen, Privilegienstrukturen und ständischen Lebensweisen einer städtischen Bürgeraristokratie einerseits, vor allem in New York, und der verschuldeten Farmer und „cowboy operators“ im Rest des Landes andererseits.

Die deutsche theoretische Soziologie freilich, die in den 1980er Jahren zur Reife kam, bezog ihren Geldbegriff nicht von Weber, sondern von Parsons und damit, über ihn, von der auf Smith zurückgehenden ökonomischen Tradition. Dies gilt nicht nur für Luhmann, sondern auch für Habermas, auch wenn oder vielleicht sogar weil dieser seine „Theorie des kommunikativen Handelns" zu einem guten Teil aus einer immanenten Kritik der Systemtheorie heraus entwickelt hat. Das Problem, wie ich es verstehe, liegt darin, dass die Habermas'sche kommunikationstheoretische Kritik an dem bei Luhmann und Parsons vorgefundenen Begriff des Steuerungsmediums dessen Geltungsanspruch für „die Funktionsbereiche der materiellen Reproduktion“ unangetastet lässt, und zwar weil diese, und allerdings nur diese, sich in der Tat „aus der Lebenswelt ausdifferenzieren“ ließen (Habermas 2014 [1987], 391). Zwar werde im ökonomischen Subsystem moderner Gesellschaften nicht wirklich gesprochen dies bleibt der Lebenswelt vorbehalten; für das, was in ihm zu sagen ist, damit es seine Funktion erfüllt, reicht aber die „Spezialsprache“ des Geldes (ibid., 388) völlig aus. Voraussetzung ist freilich, dass „Wirtschaft“ als von lebensweltlichen Bezügen gereinigtes, ohne diese auskommendes, zweckrationales wie zweckneutrales - wenn man so will: technisches - Teilsystem einer gesellschaftlichen Moderne aufgefasst werden kann, in dessen Zuständigkeitsbe- 
reich nicht gehandelt werden muss, sondern gesteuert werden kann: als berechenbarer Mechanismus der Mittel, ganz im Sinne der Standardökonomie, eingebettet zwar in einen umfassenderen, prinzipiell auch demokratisch organisierbaren Kommunikations- und Handlungszusammenhang, selbst aber darauf beschränkt, die an ihm beteiligten Akteure mit Hilfe des dazu nicht nur ausreichenden, sondern tatsächlich ideal geeigneten Steuerungsmediums Geld in wenn auch reduzierter Kommunikation allein auf die effiziente Verwendung knapper Mittel hin zu koordinieren. ${ }^{6,7}$

Aber wenn Geld nichts anderes wäre als ein neutrales Medium gesellschaftlicher Kommunikation - eine zu produktiver Koordinierung (eines speziellen Typus) menschlichen Handelns dienliche Symbolsprache - dann könnte, ja müsste man erwarten, dass der Euro nach mehr als einem Jahrzehnt seine Benutzer in einer geteilten Identität vereinigt hätte. So wie die D-Mark angeblich einen „DM-Nationalismus“ hervorgebracht hatte (Jürgen Habermas in der ZEIT vom 30. März 1990), so hätte der Euro einen Europa-Patriotismus erzeugen müssen, und genau das haben seine Erfinder auch erwartet. 1999 erklärte Jean Claude

\footnotetext{
${ }^{6}$ Zur Behandlung des Geldes bei Habermas siehe auch Dodd (1994).

${ }^{7}$ Damit ergeben sich weitreichende theoretische Konsequenzen. Die partielle Kooptation der System- in die Gesellschaftstheorie - die Anerkennung eines technokratisch-effizienztheoretischen Herrschaftsanspruchs, nicht für die Gesellschaft insgesamt, aber wohl für einen ihrer Teilbereiche, etwa nach dem Modell der Konzession eines begrenzten Zuständigkeitsbereichs an die klassische Mechanik durch die Relativitätstheorie - entpolitisiert die Wirtschaft und legt sie als Handlungssystem eindimensional auf Effizienz fest, als Preis für die Einschleusung eines Politisierungsspielraums in eine postmaterialistische Theorie der "Moderne“. Die Grundeinsicht der politischen Ökonomie, nämlich dass die effizienztheoretisch als solche daherkommenden ökonomischen Naturgesetze nichts anderes sind als Projektionen gesellschaftlicher Machtverhältnisse, die sich ideologisch als technische Zwänge abbilden, gerät in Vergessenheit. Als Folge hört Wirtschaft auf, kapitalistische Wirtschaft zu sein, und wird zu Wirtschaft als solcher, pure and simple; zugleich wird der gesellschaftliche Kampf gegen den Kapitalismus durch einen politisch-rechtlichen Kampf für Demokratie abgelöst. In der Geldtheorie geht zugunsten der Funktion des Geldes als Kommunikationsmedium der Gedanke an die hinter dem zirkulierenden Geld liegende Geldordnung im Sinne Webers und damit an die politische Rolle im Unterschied zur technischen Funktion des Geldes verloren. Dasselbe gilt für die Erkenntnis, dass Geldordnungen als politisch-ökonomische Institutionen zunächst machtund erst dann marktkonform sind und sein müssen, also im Regelfall Schlagseite in Richtung eines herrschenden Interesses aufweisen - das also, mit Schattschneider (s.o., Fußnote 5), die Sprache des Geldes ebenso wie der Gesang des „himmlischen Chores“ der pluralistischen Demokratie immer einen Akzent hat, und normalerweise denselben wie diese.
} 
Juncker, damals noch als luxemburgischer Ministerpräsident führender Steuerberater multinationaler Großunternehmen, es werde sich, wenn die Bürger mit dem Jahresbeginn 2002 erst einmal die neuen Banknoten und Münzen in Händen hielten, „bald ein neues Wir-Gefühl (bilden): wir Europäer“. ${ }^{8}$ Und im selben Jahr war von Helmut Kohl, damals schon Altbundeskanzler, zu hören, der Euro werde eine „europäische Identität" schaffen, und es werde „höchstens fünf Jahre dauern, bis Großbritannien mit in die Währungsunion komme, unmittelbar gefolgt von der Schweiz" (Hank 2015). Eine Stufe tiefer wurde der Euro in Zeitungsanzeigen mit Bildern junger, einander völkerverbindend in die Augen schauender Reiseeuropäerinnen und -europäer beworben, die sich strahlend gegenseitig vorrechneten, wie viel Geld sie wegen der entfallenen Wechselgebühren und Kursverluste auf ihren Reisen zueinander gespart hatten - Identitäts- und Effizienztheorie in einem!

Stattdessen hat der Euro Europa gespalten. Die Bilanz der Währungsunion als Motor einer ever closer union among the peoples of Europe ist verheerend. Norwegen und die Schweiz werden der EU auf lange Zeit nicht beitreten, auch wegen der damit verbundenen Beitrittspflicht zur Währungsunion. Großbritannien ist nicht nur nicht der Währungsunion beigetreten, sondern erwägt nunmehr sogar den Austritt aus der Europäischen Union. Schweden und Dänemark, die der Währungsunion irgendwann später beitreten sollten, werden dies gewiss nicht tun. Auch die Währungsunion selber ist gespalten: zwischen Über-

\footnotetext{
${ }^{8}$ http://www.spiegel.de/spiegel/print/d-15317086.html. Junckers Identitätstheorie verbindet sich zwanglos mit einer das von inm betriebene europapolitische social engineering informierenden Kognitionstheorie, wie sie in der folgenden, die Praxeologie des „permissiven Konsens“ glänzend zusammenfassenden Vorgehensbeschreibung zum Ausdruck kommt: „Wir beschließen etwas, stellen das dann in den Raum und warten einige Zeit ab, was passiert. Wenn es dann kein großes Geschrei gibt und keine Aufstände, weil die meisten gar nicht begreifen, was da beschlossen wurde, dann machen wir weiter - Schritt für Schritt, bis es kein Zurück mehr gibt" (I.c.; Hervorhebung von mir, WS). Was die dem unterliegende praktische Ethik angeht, so erinnert man sich an Junckers Maxime als Vorsitzender der Euro(-bankenrettungs-)gruppe: „Wenn es ernst wird, muss man lügen" (2011). Juncker wurde 2014 unter dem Beifall aller rechtdenkenden Europäer zum Präsidenten der EU-Kommission gewählt - alles andere hätte, laut Jürgen Habermas, „Europa ... direkt ins Herz getroffen“ (Frankfurter Allgemeine Zeitung vom 29. Mai 2015).
} 
schuss- und Defizitländern, Nord und Süd, Deutschland und dem Rest. Niemals seit Kriegs-

ende standen sich die Völker Westeuropas so feindselig gegenüber wie heute, und niemals war der erreichte Stand der europäischen Einigung so gefährdet: keiner würde mehr den Versuch wagen, auch nur kleinste zusätzliche Integrationsschritte durch die dazu nötigen Volksabstimmungen in Ländern wie Frankreich, den Niederlanden und Dänemark zu schleusen. Was speziell Deutschland angeht, so hat die Währungsunion alle deutschen wie nichtdeutschen Hoffnungen auf ein europäisches Deutschland - auf eine Lösung des deutschen Identitäts- wie Hegemonieproblems auf dem Weg über die europäische Integration - zerschlagen. An ihre Stelle ist die Furcht vor einem deutschen Europa getreten, in Europa, aber auch in Deutschland selbst. Als Folge werden in Südeuropa heute Wahlkämpfe vor allem gegen Deutschland und seine Kanzlerin ausgetragen und gewonnen, und Abbildungen von Merkel und Schäuble mit Hakenkreuzbinden sind zur Massenware geworden, nicht nur in Griechenland und Italien, sondern selbst in Frankreich. ${ }^{9}$ Dass Deutschland sich heute in Griechenland, aber auch in Italien zunehmend Forderungen nach Reparationen für den Zweiten Weltkrieg gegenübersieht, ist nur ein Zeichen dafür, wie fundamental seine Nachkriegspolitik der Selbsteuropäisierung mit dem Übergang zur Währungsunion gescheitert ist. ${ }^{10}$

\footnotetext{
${ }^{9}$ Zu Griechenland siehe die Karikatur in der Parteizeitung von Syriza, Avgi, vom 8. Februar 2015, also kurz nach dem Wahlsieg, die Schäuble in Wehrmachtsuniform zeigt und inm unter der Überschrift "Die Verhandlung hat begonnen“ folgende Worte in den Mund legt: „Wir bestehen darauf, Seife aus Eurem Fett zu machen," sowie „Wir diskutieren nur über Düngemittel aus Eurer Asche."

${ }^{10}$ Dabei geht es nicht ohne persönliche Tragödien ab. So wurde ausgerechnet Schäuble, der jahrzehntelange deutsche Champion eines „Kerneuropa“ bzw. eines „Europa der zwei Geschwindigkeiten", mit Deutschland und Frankreich als untrennbar geeintes Zentrum, Mitte April 2015 von der französischen Regierungspartei einer "unerträglichen und inakzeptablen Frankreichfeindlichkeit" beschuldigt, die sich daran zeige, dass er Frankreich „unter Vormundschaft" stellen wolle (dies und das Folgende nach Frankfurter Allgemeine Zeitung, 18. April 2015). Die Angriffe waren eine Antwort auf Äußerungen Schäubles in Washington mit dem Tenor, „es wäre besser für Frankreich, wenn es zu Reformen gezwungen würde ... aber das ist schwierig, so ist die Demokratie" - Äußerungen, die zum Commonsense jedes deutschen Finanzministers, egal von welcher Partei, gehören. Berichtet wurde, dass der Parteivorsitzende für den im Juni angesetzten Parteitag der PS an einem Aufruf „zur Konfrontation mit der europäischen Rechten“ arbeite, insbesondere „mit der CDU/CSU“. Die französische Linkspartei verlangte, „das sich Schäuble , beim französischen Volk“
} 
Wer verstehen will, wieso eine Institution wie die Währungsunion einen derartigen

Totalschaden anrichten konnte, braucht einen Geldbegriff, der über den der ökonomischen Tradition und der von dieser informierten theoretischen Soziologie hinausgeht. Die in der Währungsunion und um sie herum aufgebrochenen Konflikte sind, so meine These, nur mit Hilfe einer Theorie der politischen Ökonomie zu entschlüsseln, die Geld nicht nur als effizientes und integratives Zeichensystem zur Symbolisierung von Leistungspflichten und ansprüchen, sondern auch im Sinne Webers als Hervorbringung eines Herrschaftsverbandes und damit als umkämpfte und bestreitbare Institution mit konfliktträchtigen Verteilungseffekten aufzufassen vermag.

\section{One size does not fit all}

Erste sachdienliche Hinweise, warum die Währungsunion Europa spaltet statt es zu einigen, liefert die Literatur über unterschiedliche Ausprägungen des Kapitalismus, soweit sie historisch-institutionalistischer und nicht effizienztheoretischer Art ist. ${ }^{11}$ Die in der Währungsunion zusammengefassten nationalen Gesellschaften haben im Zuge ihrer modernen Entwicklung die kritische Schnittstelle zwischen sozialem Leben und kapitalistischem Wirtschaften jeweils anders ausgestaltet. Eine zentrale Rolle in den so konstituierten nationalen politischen Ökonomien spielt deren Geldordnung. ${ }^{12}$ Die Währungsunion kann als - wie auch im-

entschuldigen müsse. Seine Äußerungen zeigten, die neue deutsche Arroganz'. Deutschland dominiere Europa..." usw.

${ }^{11}$ Zu dieser Unterscheidung siehe Streeck (2011).

${ }^{12}$ Wie Scharpf kürzlich in kritischer Diskussion der Habermas'schen Integrationstheorie hervorgehoben hat, gehören auch die Institutionen der politischen Ökonomie und nicht nur die liberalen Garantien von Freiheit und Gleichheit zu den national erkämpften historischen Errungenschaften, die nicht ohne weiteres auf supranationaler Ebene vereinheitlicht oder zugunsten supranationaler Einheitslösungen abgeschafft werden können. Jeder, der die jahrzehntelangen fruchtlosen Auseinandersetzungen zwischen den europäischen Gewerkschaften über die richtige Form der Mitbestimmung in großen und kleinen Unternehmen verfolgt hat, weiß das. Siehe Scharpf (2015) und Habermas (2014). 
mer motivierter - Versuch verstanden werden, die an ihre jeweilige institutionelle und politische Umgebung angepassten nationalen Geldordnungen durch eine für alle beteiligten Gesellschaften gleichermaßen geltende supranationale Geldordnung zu ersetzen und so den nationalen politischen Ökonomien ein neues, neoliberales Geld zu implantieren, das sich dann den zu ihm passenden institutionellen Kontext erzwingt.

Moderne Geldordnungen, -traditionen und -praktiken sind nationalstaatlich eingebettet (Knapp 1905) und können sich grundsätzlich von Staat zu Staat unterscheiden. Im Fall der Währungsunion reicht es aber aus, idealtypisch zwischen den Ländern des Mittelmeerraums und denen Nordeuropas, insbesondere Deutschland, zu unterscheiden. ${ }^{13}$ Der europäische Süden hat einen Kapitalismustyp hervorgebracht, bei dem Wachstum vor allem von der Binnennachfrage getrieben wird, notfalls unterstützt durch Inflation, die wiederum durch einen defizitären Staatshaushalt oder durch hohe Beschäftigungssicherheit bei umfangreicher Beschäftigung im öffentlichen Sektor gestärkte Gewerkschaften angeschoben wird. Inflation erleichtert auch die Aufnahme von Staatsschulden, weil sie diese laufend entwertet. Unterstützend wirkt ein stark reguliertes, oft staatliches oder halbstaatliches nationales Bankensystem. Alle zusammen ermöglichen es, die Interessen von Arbeitnehmern und typisch binnenwirtschaftlich und kleinbetrieblich operierenden Arbeitgebern mehr oder weniger gut zum Ausgleich zu bringen. Der Preis für den so hergestellten sozialen Frieden ist ein Verlust an internationaler Wettbewerbsfähigkeit im Vergleich zu Hartwährungsländern, der aber bei monetärer Souveränität durch periodische Abwertung der nationalen Währung auf Kosten ausländischer Exporteure aufgefangen werden kann.

\footnotetext{
${ }^{13}$ Zum Folgenden siehe unter anderen Armingeon und Baccaro (2012), Baccaro und Benassi (2014), Blankart (2013), Hall (2012), Hancké (2013), Höpner und Lutter (2014), Iversen und Soskice (2013) und Johnston und Regan (2014).
} 
Die nördlichen in der Währungsunion zusammengeschlossenen Volkswirtschaften funktionieren anders. Ihr Wachstum beziehen sie aus Exporterfolgen und sind deshalb inflationsavers. Letzteres gilt, gelegentlicher „keynesianischer“ Rhetorik zum Trotz, auch für die Arbeitnehmer und ihre Gewerkschaften, zumal heute, da Kostensteigerungen mehr denn je zur Umsiedlung industrieller Produktionsstätten führen können. Eine Abwertungsoption brauchen derartige Volkswirtschaften nicht unbedingt; was Deutschland angeht, so ist es seit den 1970er Jahren mit immer neuen Aufwertungen seiner Währung durch Aufwertung seiner Produkte und Abwanderung aus preiskompetitiven in qualitätskompetitive Märkte gut zurechtgekommen. Anders als die Mittelmeerländer sind Länder wie Deutschland auf eine harte Währung eingestellt. Das macht sie nicht nur inflations-, sondern auch verschuldungsavers, obwohl die Zinsen, die sie zu zahlen haben, vergleichsweise niedrig sind. Dass sie ohne eine lockere Geldpolitik auskommen, enthebt sie des Weiteren der Notwendigkeit, das Risiko von Blasenbildungen in Vermögensmärkten auf sich nehmen zu müssen, und kommt überdies ihren Sparern zugute, von denen es viele gibt und deren Stimmen deshalb politisch ins Gewicht fallen. ${ }^{14}$

Es ist wichtig zu betonen, dass keine der verschiedenen Ausformungen des Grenzbereichs zwischen Kapitalismus und Gesellschaft den anderen per se moralisch überlegen ist. Jede Einbettung des Kapitalismus in die Gesellschaft und jeder Versuch einer Einpassung seiner Logik in die des sozialen Lebens ist unvermeidlich unvollkommen und "unsauber", improvisiert und kompromittiert und für keine Seite jemals voll zufriedenstellend. Dessen ungeachtet halten die entschiedensten Parteigänger der jeweiligen nationalen Kapitalismusmodelle alle alternativen Modelle für minderwertig, ihr eigenes dagegen für einzigrichtig

\footnotetext{
${ }^{14}$ „Erst sparen, dann kaufen“ ist eine zutreffend zugespitzte Zusammenfassung der traditionellen deutschen, von einem komplexen Bündel komplementärer politisch-ökonomischer Institutionen unterstützten Wirtschaftsweise und Wirtschaftskultur. Siehe kürzlich Mertens (2014).
} 
und einzigvernünftig, was darauf zurückzuführen ist, dass bei einem Konflikt der Modelle nicht nur der materielle Lebensunterhalt auf dem Spiel steht, sondern auch die jeweils eingelebte moral economy. Kultureller Chauvinismus dieser Art bringt im Norden Europas das Klischee von den „faulen Griechen“ hervor und im Süden die Vorstellung von den „kalten Deutschen“, die „leben um zu arbeiten, statt zu arbeiten um zu leben“, verbunden mit der dringenden Aufforderung an die jeweils andere Seite, ihre Fehler zu erkennen und sich zu bessern. Aufgrund derselben Perspektivenverzerrung können Deutsche, die von den Griechen verlangen, ihre Wirtschaft, Gesellschaft und sich selbst zu „reformieren“, um Korruption und Verschwendung ein Ende zu bereiten, nur ausnahmsweise auf den Gedanken kommen, dass sie diese damit letztlich auffordern, ihre altmodische, lokal eingebettete Korruption durch die moderne, global entbettete Korruption a la Goldmann Sachs abzulösen, wie sie dem finanzialisierten Kapitalismus der Gegenwart eigen ist. ${ }^{15}$

Für unterschiedliche Gesellschaftsverträge ausgelegte Geldordnungen können mehr oder weniger friedlich koexistieren, wenn sie über ein Mindestmaß an Souveränität verfügen und ihre Währungen zum Ausgleich ihrer unterschiedlichen Wettbewerbsfähigkeit gegeneinander neubewerten können. Ein einheitliches Geldregime dagegen für so verschiedene Volkswirtschaften wie die nordeuropäischen Angebots- und die südeuropäischen Nachfrageökonomien kann nicht auf alle gleich gut passen. Die Folge ist, dass in ihm qualitativhorizontale Diversität in quantitativ-vertikale Ungleichheit transformiert wird. Werden poli-

\footnotetext{
${ }^{15} \mathrm{Ob}$ und in welcher Weise es wünschenswert wäre, Gesellschaften wie die griechische oder spanische im Sinne einer Befreiung von ihren „feudalen Fesseln“ (Hirschman 1982) zu "modernisieren“, lasse ich aus zwei Gründen dahingestellt sein: weil dies ohnehin nicht als „Durchregieren“ von außen geschehen kann und weil es eben nicht nur einen Weg gibt, Kapitalismus und Gesellschaft (zeitweise) miteinander zu vereinbaren. Mehr noch als die Einzelstaaten der USA könnten und sollten die alten Nationalstaaten Europas als "laboratories of democracy" (Lewis Brandeis in New State Ice Co. vs. Liebman, 1932) dienen, wobei Demokratie nicht nur die institutionellen Formalien kollektiver Diskussion und Willensbildung einschließt, sondern auch die jeweilige, immer nur vorläufige Ausgestaltung der Konfliktzone zwischen sozialem Leben und kapitalistischem Wirtschaften.
} 
tisch unterschiedlich ausgestaltete Volkswirtschaften in einer Währungsunion zusammengefasst, geraten die von dieser heruntergewerteten Wirtschaftsgesellschaften unter Druck, ihre Produktionsweise und ihren an diese angepassten Gesellschaftsvertrag nach dem Vorbild der von der Währungsunion privilegierten Länder zu "reformieren". Nur wenn sie dies können und wollen, wenn also die einheitliche Geldordnung eine einheitliche Kapitalismusordnung nach sich zieht, kann eine gesellschaftenübergreifende Währungsunion konfliktfrei funktionieren.

Dass ein einheitliches Geldregime für unterschiedliche Volkswirtschaften diese unterschiedlich bevorteilen und dadurch selektiv unter Anpassungszwang setzen kann, prägte schon die mit der Währungsunion verfolgten strategischen Ziele und die um ihretwillen eingegangenen Kompromisse. Von Anfang an war die gemeinsame Währung eine in sich widersprüchliche und konfliktträchtige Konstruktion. Frankreich und andere europäische Länder, insbesondere Italien, waren es leid, der Hartwährungs-Zinspolitik der Bundesbank, die unter den Bedingungen der Kapitalverkehrsfreiheit im sich finanzialisierenden Kapitalismus de facto zur Zentralbank Europas geworden war, folgen zu müssen. Ebenso zuwider war ihnen, vor allem den Franzosen, die periodisch sich aufdrängende, als nationale Demütigung empfundene Notwendigkeit, zur Erhaltung ihrer Wettbewerbsfähigkeit ihre Währung gegen die deutsche abzuwerten. Von einer Ersetzung der Bundesbank durch eine europäische Zentralbank erhofften sie sich, einen Teil ihrer an Deutschland verlorenen geldpolitischen Souveränität zurückzugewinnen und zugleich die Geldpolitik in Europa weniger stabilitätsorientiert gestalten und stärker auf politische Ziele wie Vollbeschäftigung ausrichten zu können. Allerdings setzten Mitterand und sein Finanzminister Delors, später als Chef der Europäischen Kommission einer der Architekten des Euro, wie auch die Banca d'Italia, die italienische Zentralbank, zugleich auch darauf, mit Hilfe der Währungsunion und des durch sie bewirkten 
Ausschlusses der Abwertungsoption ihre jeweiligen kommunistischen Parteien und Gewerkschaften mittels einer härteren oder gar harten Währung zu zwingen, ihre politischökonomischen Asoirationen aufzugeben.

In Deutschland waren die Bundesbank und die überwiegend ordoliberal und monetaristisch orientierte Ökonomenzunft gegen die Währungsunion, weil sie eine Untergrabung der deutschen „Stabilitätskultur“ befürchteten. Auch der Bundeskanzler hätte es vorgezogen, wenn der Währungsunion eine politische Union - am besten natürlich mit einer deutschen Wirtschaftspolitik - vorausgegangen wäre. Seine europäischen Partner aber hatten die gemeinsame Währung ja nicht verlangt, um ihre Souveränität noch weiter zu verlieren, sondern um sie im Gegenteil wenigstens teilweise zurückzugewinnen. Aus Furcht um die Wiedervereinigung gab Kohl nach, wohl in der Erwartung, dass die Währungsunion die politische Union irgendwie nach sich ziehen würde - eine Erwartung, die heute allenfalls noch von der deutschen Euro-Linken als letzten Anhängern der neofunktionalistischen Integrationstheorie geteilt wird. Als wichtige Kräfte in Kohls politischem Lager drohten, ihm die Gefolgschaft zu verweigern, überwand er ihren Widerstand, indem er durchsetzte, dass das gemeinsame europäische Geldregime genau dem deutschen Modell folgte, mit der Europäischen Zentralbank als vergrößerter Kopie der deutschen Bundesbank.

Damit waren die Konflikte der folgenden Jahre vorgezeichnet. Der Slogan, mit dem die deutsche Regierung ihren skeptischen Wählern den Euro nahebrachte, war „Der Euro stabil wie die Mark“. Deutschlands Partnerstaaten unterschrieben den Vertrag dennoch, vermutlich darauf setzend, dass es ihnen gelingen würde, ihn später unter dem Druck wirtschaftlicher „Realitäten“ umzuschreiben, wenn nicht auf dem Papier, dann in der Praxis. Es half, dass die 1990er Jahre eine Epoche waren, in der, ausgehend von den USA, alle westlichen OECD-Länder eine Politik der fiskalischen Konsolidierung verfolgten, im Zusammenhang 
der „Finanzialisierung“ des Kapitalismus und des Übergangs zu einer neoliberalen, nichtKeynesianischen Wirtschaftspolitik (Streeck 2015b). Sich auf einen Schuldendeckel von sechzig Prozent des Sozialprodukts und Haushaltsdefizite von nicht mehr als drei Prozent zu verpflichten entsprach dem Geist der Zeit, und „die Märkte“ hätten Mittel und Wege gekannt, ein Land, dass sich verweigert hätte, ihr Missfallen spüren zu lassen.

Die in der Währungsunion angelegten ungleichen Begünstigungs- und Verteilungseffekte machten sich schon früh bemerkbar. Heute ist es Deutschland, zusammen vor allem mit den Niederlanden, Österreich und Finnland, das von der gemeinsamen Währung profitiert. Aber dies ist erst seit 2008 so. In der Frühphase der Währungsunion machte die einheitliche Geldpolitik Deutschland zum „kranken Mann Europas“. Damals lag der Zinssatz für den Euro oberhalb der deutschen Inflationsrate, weshalb die institutionell und wirtschaftlich inflationsunfähige deutsche Ökonomie unter zu hohen Realzinsen litt. Gleichzeitig verzeichnete der Mittelmeerraum Inflationsraten oberhalb des gemeinsamen Zinssatzes und kam so in den Genuss von negativen Realzinsen (Scharpf 2013). Zusätzlich sanken die Kosten staatlicher Kreditaufnahme im Süden Europas dramatisch, zum guten Teil wegen der auch von der Europäischen Kommission inspirierten Annahme der „Kapitalmärkte“, dass die gemeinsame Währung, Verträge hin oder her, eine wie immer geartete gemeinsame, oder gar deutsche, Verantwortung für die Zahlungsfähigkeit der Mitgliedsländer beinhaltete. Ergebnis waren ein Boom im Süden und Stagnation, bei hoher Arbeitslosigkeit und zunehmender Staatsverschuldung, in Deutschland.

Dies änderte sich 2008 mit dem Ende der Kreditschwemme, also dem Platzen der IIlusionen der Finanzmärkte über die deutsche oder auch europäische Bereitschaft, als lender of last resort die Schulden des Südens zu übernehmen, und dem gleichzeitigen Rückgang des Zinssatzes auf nahezu null Prozent. Dass die gemeinsame Währung nun die Deutschen be- 
günstigte, lag aber vor allem an der noch in den 1990er Jahren beklagten „Über-

Industrialisierung" ihrer Volkswirtschaft, die sie gegenüber der Fiskalkrise und dem Zusammenbruch des Kredits unempfindlicher machte als die stärker von ihrer Binnennachfrage abhängigen EMU-Länder, auch weil sie es ihr ermöglichte, sich noch mehr als bisher auf die Bedienung globaler Märkte für qualitätskompetitive Industriegüter zu verlegen. Hinzu kam die Unterbewertung des Euro als Währung für das deutsche im Unterschied zum europäischen Wirtschaftsgebiet. ${ }^{16}$ So wurde Deutschland, ungeplant und ebenso unwillig wie umstritten, bis auf weiteres zur europäischen Hegemonialmacht. Zugleich begann wegen der unterschiedlichen Kompatibilität der politischen Ökonomien der Mitgliedsländer mit dem einheitlichen Geldregime ein immer härteres Tauziehen zwischen Nord und Süd, bei dem es im Wesentlichen um drei Themen ging und geht: die Auslegung und, vielleicht, Revision der vertraglich vereinbarten gemeinsamen Geldordnung; die Pflicht der Mitgliedsländer zu institutionellen „Reformen“ zwecks Anpassung entweder des Südens an den Norden oder umgekehrt; und, bei fortdauernder Disparität der Einkommens- und Lebensverhältnisse, die Art und das Ausmaß der Ausgleichszahlungen des Nordens an den Süden.

\section{Kampf zweier Linien}

Bevor ich im Folgenden nacheinander auf die drei Themen eingehe, liegt mir daran zu betonen, dass keins davon durch Rettungsoperationen der gegenwärtig betriebenen Art, und seien sie noch so erfolgreich, zu erledigen sein wird. ${ }^{17}$ Tatsächlich geht es bei ihnen um un-

\footnotetext{
${ }^{16}$ Im Jahr 2013 war der Euro laut Morgan Stanley bei einem Wechselkurs von 1,36 US-Dollar für Deutschland um 13 Prozent unter- und für Italien und Griechenland um 12 bzw. 24 Prozent überbewertet (zitiert bei Streeck und Elsässer 2014, 8).

${ }^{17}$ Es gehört zu den erstaunlichen Fehlleistungen der gegenwärtigen politischen Diskussion, insbesondere in Deutschland, dass die Probleme der Währungsunion als eine einzige, wenn auch schwere Einmalkrise behandelt werden, die durch möglicherweise hohe, immerhin aber nur einmal anfallende Rettungszahlungen an Banken oder Staaten oder beide ein für alle Mal überwunden werden kann.
} 
terschiedliche Manifestationen einer tiefen Spaltung der Währungsunion als politisches System, die durch deren „Rettung“ nicht beseitigt, sondern im Gegenteil erst richtig wirksam werden wird. Was die erste Ebene angeht - den Kampf um die praktische Auslegung des Vertrags von Maastricht - so stoßen südeuropäische Versuche, mit Hilfe der EZB den Euro aufzuweichen und so auf Umwegen zu Inflation, Schuldenfinanzierung des Staates und Währungsabwertung zurückzufinden, auf den Unwillen der Nordländer, die nicht durch Mehrheitsbeschlüsse als Ersatzkreditgeber herangezogen werden und für jene Vorabinjektionen von Geld bürgen wollen, ohne die die Volkswirtschaften ihrer südländischen Partner in ihrer gegenwärtigen Gestalt nicht funktionieren könnten. Insoweit spielt sich die Innenpolitik der EWU schon heute zwischen Allianzen von Mitgliedstaaten ab, die das gemeinsame Geldregime in entgegengesetzte Richtungen zu ziehen suchen, die einen nach Süden und die anderen (zurück) nach Norden. Jeder der beiden Blöcke kann in seiner gegenwärtigen politischökonomischen Gestalt mit dem anderen auf Dauer nur auskommen, wenn er die Deutungshoheit über das Geldregime erobert. Ohne den anderen auskommen will aber keiner: Während die Nordeuropäer die festen Wechselkurse für ihre Exportindustrie schätzen, wollen die Südeuropäer niedrige Zinsen und sind dafür bereit, vertraglich festgelegte Schuldendeckel und Defizitgrenzen hinzunehmen, in der Hoffnung, dass im Notfall Partnerländer leichter politisch-diplomatisch unter Druck zu setzen oder zu beschwichtigen sind als Finanzmärkte. In der Auseinandersetzung um die „richtige“ Deutung der Währungsunion haben die derzeitige deutsche Regierung und ihre nordeuropäischen Verbündeten noch immer die Oberhand, zumindest solange der Süden auf ihre Rettungsmilliarden angewiesen ist. Bliebe dies so, hätten die Südländer am Ende keine andere Wahl, als ihre politischen und wirtschaftlichen Institutionen an das dann vom Norden autoritativ als neoliberal interpretierte 
europäische Geldregime anzupassen ${ }^{18}$ - mit ungewissem Ausgang und, selbst wenn alles gutginge, einer langen Übergangszeit voll politischer Unruhe und wirtschaftlicher Ungewissheit. So würden beispielsweise die südlichen Mitgliedsländer ein „flexibles“ nordeuropäisches Arbeitsmarktregime zu akzeptieren haben, während bei einem entgegengesetzten Ausgang des europäischen Verfassungskampfes die Deutschen ihr in den Augen anderer Länder destruktives Sparverhalten und ihre egoistische Exportorientierung aufgeben müssten.

Damit würde sich der „Marktkampf“ auf die nächstuntere Ebene verlagern, auf der es um die den Mitgliedsländern abverlangten institutionellen „Reformen“ geht. Für den Norden sprächen hier neben seiner wirtschaftlichen Übermacht der Wortlaut der Verträge und die von der EZB ausgehenden Reform- und Konsolidierungsauflagen, für den Süden unter anderem seine Mehrheit in den Gremien von EWU und EZB sowie, vielleicht, das europäische Harmoniebedürfnis der deutschen politischen Klasse. Politisch ist allerdings gerade im Kernbereich der politisch-ökonomischen Institutionen der an der Währungsunion beteiligten Nationalstaaten mit heftigem, demokratisch legitimiertem Widerstand gegen „Reformen“ und als Folge mit einem lange andauernden, wenn nicht dauerhaften Nebeneinander inkompatibler politisch-ökonomischer Institutionen unterhalb des gemeinsamen Währungsregimes zu rechnen. Ebenso wie die Südländer ihren Kündigungsschutz und ihre gesicherte Beschäftigung im öffentlichen Sektor werden verteidigen müssen, können in den Nordländern die Beschäftigten in der Exportwirtschaft nicht auf ihre „betrieblichen Bündnisse“ verzichten oder sich auf Lohnabschlüsse einlassen, die die Wettbewerbsfähigkeit ihrer Unternehmen und damit ihre Arbeitsplätze gefährden würden. Weder würde der Süden seine Produktivität

\footnotetext{
${ }^{18}$ Und das aktuelle quantitative easing der EZB wäre dann doch kein Regimewechsel in Richtung Süden, sondern nur eine zeitweilige Absicherung für Regierungen, die im Gegenzug „Reformen“ zu liefern hätten. Soweit bei jemandem wie Draghi politische Überzeugungen zu erkennen sind, sprechen sie für diese Vermutung und nicht für einen kalten Regimewechsel in Richtung Süden.
} 
noch der Norden seine Kosten so erhöhen können, dass es zu Konvergenz käme. ${ }^{19}$ Der Kampf zweier Linien ginge weiter, während die Exportanteile und -überschüsse im Norden zunähmen und der Deflations- und Rationalisierungsdruck im Süden anhielte.

Resultat wäre, und damit sind wir bei der dritten Konfliktebene, ein Dauerkonflikt zwischen den Mitgliedsländern der Währungsunion über die europäische, oder genauer: euro-europäische, Finanzverfassung, analog der unendlichen Auseinandersetzung in Deutschland über den Länderfinanzausgleich, in der EWU allerdings als Kampf zwischen souveränen Staaten, ohne das Dach einer gemeinsamen demokratischen Verfassung, ohne ein auch nur annähernd so dichtes Netz von gemeinsamen Institutionen wie in einem Nationalstaat und nicht innerhalb einer einzigen, mehr oder weniger einheitlichen Wirtschaftsgesellschaft, sondern zwischen unterschiedlichen national institutionalisierten Kapitalismus-Varianten und im Medium der emotional gefährlich aufladbaren internationalen Beziehungen. Die Beträge, um die es dabei ginge, wären erheblich, und sie wären laufend fällig - übrigens auch dann, wenn sich die geforderten neoliberalen „Strukturreformen“ im Süden tatsächlich durchsetzen ließen und die betroffenen Länder nach einer Deflation um 20 bis 30 Prozent auf Aufholjagd gingen. Dass sie dabei ohne Hilfe dauerhaft schneller wachsen würden als die Länder des Nordens, können sich nämlich nur Ökonomen vorstellen. ${ }^{20}$

\footnotetext{
${ }^{19}$ In Anerkennung dessen hat die deutsche Linke (Lafontaine, Flassbeck) vor einiger Zeit ihre jahrelang erhobene Forderung zurückgezogen, dass die deutschen Gewerkschaften durch eine aggressive Lohnpolitik die Wettbewerbsvorteile der deutschen Volkswirtschaft in der Währungsunion abbauen und so durch Anpassung an die Wirtschaftsweise des Südens die für die einheitliche Währung nötige interne wirtschaftliche Konvergenz herbeiführen sollen. Die nunmehr erhobene Forderung nach Auflösung der Währungsunion in ihrer derzeitigen Form (Flassbeck und Lapavitsas 2015) ist die logische Konsequenz.

${ }^{20}$ Vergleichbar dem Glauben der Ökonomen an Konvergenz durch Reform ist der Glaube der eurofreundlichen Linken an Konvergenz durch Entschuldung: beides gleich unrealistisch und nur als rhetorisches Mittel zur Immunisierung einer ideologischen Utopie gegen empirisch basierte Zweifel zu verstehen.
} 
Wie hoch die vom Norden zu leistenden Ausgleichszahlungen wären, lässt sich nicht mit Sicherheit sagen; wissen kann man aber, dass sie nicht reichen würden, um das NordSüd-Gefälle einzuebnen (zum Folgenden siehe Streeck und Elsässer 2014). Rechnet man ein, dass es nicht nur um Griechenland ginge, sondern auch um Portugal und Spanien und möglicherweise den gesamten Mittelmeerraum, ${ }^{21}$ dann müssten die in einer "Transferunion" vom Norden zu leistenden Hilfs- und Ausgleichszahlungen proportional mindestens so hoch sein wie die jährlichen Mittel, die Deutschland seit seiner Währungsunion mit der ehemaligen DDR seinen "Neuen Ländern" und die Italien seit dem Ende des Zweiten Weltkriegs mehr oder weniger regelmäßig dem Mezzogiorno zukommen lässt: rund vier Prozent des Sozialprodukts, in beiden Fällen mit dem bescheidenen Ergebnis, dass die Einkommenslücke zwischen reichen und armen Regionen, statt sich zu schließen, lediglich nicht größer wurde (Streeck und Elsässer 2014, 19). Was den Haushalt der EU angeht, so müsste dieser mindestens um dreihundert Prozent wachsen, von einem auf vier Prozent des Sozialprodukts aller EU-Länder. Ein Land, das seinen Beitrag an die EU um drei Prozent seines Sozialprodukts aufzustocken hätte - und um mehr, wenn einzelne Länder sich nicht beteiligen wollten oder könnten - müsste bei einem Staatsanteil von 40 Prozent an seiner Wirtschaftsleistung insgesamt siebeneinhalb Prozent seiner öffentlichen Ausgaben nach Brüssel überweisen. In Deutschland, wo der Bundeshaushalt etwa die Hälfte der öffentlichen Ausgaben ausmacht, würde er um das doppelte, rund 15 Prozent, zunehmen müssen, soweit die zusätzlichen Transfers an die EU nicht durch Einsparungen bestritten werden könnten - ein Anstieg, der

\footnotetext{
${ }^{21}$ Sowie perspektivisch, aus Sicht der geostrategisch orientierten und je weiter östlich, desto mehr von den USA inspirierten Beitrittspolitik der EU, um den gesamten Balkan von Serbien bis Albanien - alles potentielle und durchaus selbsterklärte Unterstützungsempfänger, die unter einer harten Währung noch weit hilfsbedürftiger wären als Griechenland, von der extremen Anfälligkeit ihrer Sozialstruktur für Klientelismus und Korruption zu schweigen. Es ist bemerkenswert, dass die Probleme und Kosten einer Südosterweiterung, wie sie nach einer allerdings unwahrscheinlichen Behebung der Krise des Mittelmeerraums, vielleicht aber auch ohne diese anstünde, von niemandem auch nur erwähnt werden. Das einschlägige Stichwort hier wäre overextension.
} 
sich nicht über Jahrzehnte verteilen würde, sondern in kürzester Frist gemeistert werden müsste, noch dazu in Zeiten niedrigen Wachstums und allgemeiner fiskalischer Konsolidierung. $^{22}$

Damit sind die Hauptkonfliktlinien jeder künftigen EWU-Innenpolitik umrissen. Über einmalige, humanitär zu begründende "Rettungszahlungen" hinaus werden Transfers innerhalb der Währungsunion politisch nur möglich sein, wenn und soweit sie die Größenordnung der seit langem bestehenden Regional- und Sozialfonds der EU nicht dramatisch überschreiten und sich glaubhaft als Hilfe zur Selbsthilfe ausweisen lassen. Laufende Umverteilung als solidarischer Ausgleich für geringere Wettbewerbsfähigkeit unter einer harten Währung wäre weder in den zu immer härterer Arbeit angehaltenen Leistungsgesellschaften des Nordens durchzusetzen noch auf die Dauer mit dem nationalen Selbstwertgefühl der Empfängerländer vereinbar. Bei Leistungen aber, die sich selber überflüssig machen sollen - wie auf nationaler Ebene in der klassischen Regionalpolitik und international in der Entwicklungspolitik ist die ungeduldige Frage der Zahler, wann sie endlich nicht mehr benötigt werden, ebenso unvermeidlich wie die Sorge, dass sie vielleicht nicht nur für investive, sondern auch teilweise oder gar ganz für konsumtive Zwecke eingesetzt werden. Damit das, was als zeitlich beschränkte Nothilfe noch legitimierbar wäre, sich in der Praxis nicht in Daueralimentation verwandelt, werden ihre Geber sie nur unter Auflagen und gegen Kontrollrechte gewähren wollen. Vor allem im Verhältnis zwischen Nationen und souveränen Staaten muss dies zu Spannungen führen, die sich unter anderem in Vorwürfen imperialistischer und demokratieuntergrabender Einmischung in innere Angelegenheiten manifestieren würden. Während die Empfängerländer über zu niedrige Zahlungen bei zu weit gehenden Forderungen nach Sou-

\footnotetext{
${ }^{22}$ Die im Süden Europas erstaunlich populäre Vorstellung, dass Deutschland irgendwie - aus Eigeninteresse oder aus Schuld- oder Solidaritätsgefühl, aus Liebe zu oder aus Angst vor Europa - die europäischen wirtschaftlichen Asymmetrien im Alleingang reparieren würde, ist noch um eine weitere Größenordnung unrealistischer.
} 
veränitätsverzicht klagen werden, werden umgekehrt die Regierungen und die Öffentlichkeiten der Geberländer die ihnen abverlangten Unterstützungsleistungen für zu hoch und die im Gegenzug gewährten Eingriffsmöglichkeiten für zu gering halten. So wird sich die zukünftige Innenpolitik der EWU im Wesentlichen um die Achse Geld gegen Kontrolle drehen und dabei vielfältige Möglichkeiten für nationalistisch-demagogische Mobilisierung auf allen beteiligten Seiten eröffnen.

\section{Eine neue Geldordnung}

Schon lange sind positive Argumente für die Beibehaltung der Währungsunion nicht mehr zu hören, weder politischer noch wirtschaftlicher Art. Der einzige Grund, den die Verteidiger des Status quo noch gegen einen Abbruch dessen ins Feld führen, was Polanyi zweifellos ein "frivoles Experiment" genannt hätte (Polanyi 1957 [1944]), ist, dass dessen Folgen zwar nicht vorhersehbar, aber jedenfalls schlimmer wären als die einer Fortsetzung der institutionalisierten Dauerkrise. ${ }^{23}$ Dahinter steht wohl auch die Angst der europäischen politischen Klasse vor der Rechnung, die ihnen die Wähler für die Leichtfertigkeit präsentieren könnten, mit der sie den Wohlstand und das friedliche Zusammenleben eines ganzen Kontinents aufs Spiel gesetzt haben.

Eigentlich könnte man erwarten, dass die möglichen Kosten eines Um- oder Rückbaus der Währungsunion nicht mehr lange als Argument für deren Fortführung ausreichen werden. Die Hoffnung im Norden, mit einer rettungspolitischen Einmalzahlung oder gar einer strukturreformerischen Einmaldeflation im Süden davonzukommen, wird ebenso schwinden wie die im Süden auf politische Daueralimentation von für ein Hartwährungsregime nicht

\footnotetext{
${ }^{23}$ Wenn man von denjenigen Befürwortern eines Festhaltens an der Währungsunion absieht, die sich von ihr einen heilsamen, wenn auch zunächst schmerzlichen Zwang für Deutschland versprechen, in sich zu gehen und seine nationalen Interessen als Verzicht auf dieselben neu zu definieren. Praktisch läuft dies freilich auf ein gefährliches Spiel mit dem anti-europäischen Feuer in dem letzten Land Europas hinaus, das noch keine rechtspopulistische Massenpartei hervorgebracht hat.
} 
geeigneten Wirtschafts- und Sozialstrukturen. Zugleich wird sich, je länger je mehr, die Hoffnung auf eine irgendwie zustande und dann zu Hilfe kommende Sofortdemokratie auf gesamteuropäischer Ebene als Illusion erweisen (Streeck 2015a). Schon gar nicht wird sich eine gesamteuropäische Demokratisierung dadurch erzwingen lassen, dass man die Dauerkrise der Währungsunion schmoren lässt, in der Hoffnung, dass irgendwann der Schmerz zuviel wird, nicht so sehr der wirtschaftliche im Süden wie der politisch-moralische im Norden, namentlich in Deutschland. Viel wahrscheinlicher als eine gemeinsame demokratische Flucht nach vorn ist, dass die nationalen Demokratien Europas in die Hände aggressivnationalistischer Parteien fallen. Als Unterstützer einer Integration durch Währungsunion blieben dann, außer den um ihre Ämter fürchtenden Politikern, nur noch die Mittelschichten des Südens, die auf den Rockschößen einer europäischen kapitalistischen Marktwirtschaft in eben jenes sozialdemokratische Konsumparadies gelangen wollen, das gerade dabei ist sich aufzulösen, sowie die Exportindustrien des Nordens, die möglichst lange den gerne auch kreditfinanzierten Konsum der Südländer ebenso mitnehmen wollen wie die Wettbewerbsvorteile einer gegenüber dem Rest der Welt unterbewerteten gesamteuropäischen Währung. Wenn aber die Konvergenz endgültig ausbleibt und der laufende Umverteilungsbedarf in seiner Gänze sichtbar wird, dürfte dies wahlpolitisch nicht mehr reichen, nicht einmal in Deutschland.

Worauf es deshalb heute ankäme, wäre, mit der Sakralisierung des Geldregimes der Währungsunion und seiner „typisch deutschen“ Aufladung mit postnationalen Heilsattributionen ${ }^{24}$ und -erwartungen zu brechen und die Währungsunion ohne die üblichen Horror-

\footnotetext{
${ }^{24}$ Wie der Behauptung, die Währungsunion sichere den Frieden in Europa und sei deshalb unentbehrlich. Der lange europäische Frieden des 20. Jahrhunderts begann 1945, die Währungsunion gab es erst 1999. Neben der Europäischen Union (mit nationalen Währungen) waren es vor allem die NATO und der Kalte Krieg, die die Länder Westeuropas, anders als in der Zwischenkriegszeit, zu gegenseitiger Friedlichkeit anhielten. Die Währungsunion dagegen hat, als sie einmal bestand, in
} 
szenarien ${ }^{25}$ als das zu diskutieren, was sie ist: eine wirtschaftliche Zweckkonstruktion, die ihr

Existenzrecht verwirkt hat, wenn sie ihren Zweck nicht erfüllt. Im letzten Teil eines vor zweieinhalb Jahren geschriebenen Buches, das über Erwarten Aufmerksamkeit gefunden hat (Streeck 2013), habe ich eher zaghaft eine Um- und Neugründung der Währungsunion in Anlehnung an das ursprüngliche, von Keynes entwickelte Modell von Bretton Woods vorgeschlagen: mit dem Euro als Anker für nationale oder auch multinationale Einzelwährungen und mit vertraglichen Vorkehrungen zum Ausgleich wirtschaftlicher Ungleichgewichte, einschließlich der Möglichkeit einer Neufestsetzung der Wechselkurse. Damit wäre der in der Währungsunion faktisch geltende Goldstandard, der die nationalen Demokratien leerlaufen lässt, ohne eine supranationale Demokratie zu befördern, aufgehoben. ${ }^{26}$ Da ich von den technischen Einzelheiten nicht genug und noch weniger verstand als heute, habe ich diesen Vorschlag nicht weiter ausgeführt - auch weil ich mir sicher war, dass die Europa regierenden Eliten ohnehin stur an ihrem Einigungsprojekt festhalten würden, wie spaltend es auch sein mochte. So ist es denn auch gekommen. Allerdings finden sich in der öffentlichen und sogar der wissenschaftlichen Diskussion mittlerweile erstaunlich viele Voten für ein flexibleres Währungsregime, das demokratische Politik in die Lage versetzen könnte, Ungleichge-

\footnotetext{
Westeuropa nicht Frieden, sondern Unfrieden gestiftet. Was den Beitrag der europäischen Einigung insgesamt zur Friedenssicherung angeht, so erscheint dieser in einem sehr anderen als dem offiziellen Licht, wenn man den Fall der Ukraine betrachtet, wo die Pläne "des Westens" für eine weitere Osterweiterung der EU den nunmehr dort bestehenden Kriegszustand nachhaltig gefördert haben.

${ }^{25}$ Als krasses Beispiel mag das unsäglich demagogische Merkel'sche Diktum gelten, das immer noch bis weit in die Reihen der Linken als Dogma rezipiert wird: „Scheitert der Euro, dann scheitert Europa" (Regierungserklärung vom 19. Mai 2010). Selbsterklärte Europhile wie Merkel verwechseln das kulturelle Erbe Europas mit den von innen zu verantwortenden politischen Fehlentscheidungen.

${ }^{26}$ Und man wäre annähernd zu dem Zustand von Anfang 1999 bis Ende 2001 zurückgekehrt, als der Euro und die nationalen Währungen der Mitgliedstaaten nebeneinander bestanden, allerdings mit festen, nicht mehr änderbaren Paritäten. Der Unterschied wäre, dass nun die Paritäten revidierbar wären, nicht durch den Devisenmarkt oder einseitiges Regierungshandeln, sondern durch ein vertraglich geordnetes Verfahren.
} 
wichte auf weniger destruktive Weise zu korrigieren als immer nur und allein durch innere Abwertung. Die dazu gemachten Vorschläge reichen von einer Rückkehr zu nationalen Währungen über die zeitweilige oder dauerhafte Einführung von Parallelwährungen mit Kapitalverkehrskontrollen bis hin zu einem doppelstöckigen Währungssystem a la Keynes. ${ }^{27}$

Dafür, ein gemeinsames Nachdenken über einen sozial-, europa- und demokratieverträglichen Um- und Neubau der Europäischen Währungsunion für dringlich zu halten, bedarf es keiner „D-Mark-Nostalgie“. Im Prinzip könnte sich das Thema auch aus dem Zusammenhang der dringend anstehenden Suche nach einer besseren als der derzeitigen Geldordnung auf globaler Ebene ergeben - einer Geldordnung, die seit der endgültigen Demontage des Regimes von Bretton Woods Anfang der 1970er Jahre immer dysfunktionaler geworden ist, bis sie 2008 die Weltwirtschaft beinah zum Einsturz brachte. Das Scheitern des Euro ist nur eine unter vielen anderen Entwicklungen, die die aus der eigentümlich befriedeten Nachkriegszeit stammende Vorstellung immer deutlicher als zeitbedingte Illusion erkennen lassen, wonach ein für alle Mal unproblematisch feststeht, was Geld ist und wie es zu regieren wäre. Diskussionen über ein globales Währungs- und Finanzregime, das flexibel genug wäre, um den Entwicklungsbedingungen und -notwendigkeiten aller an der Weltwirtschaft beteiligten Gesellschaften gerecht zu werden, ohne Abwertungswettläufe und kompetitive Geld- und Schuldenproduktion mitsamt den von ihnen geschürten geostrategischen Rivalitäten, wären mehr als nötig. Sie könnten übrigens an die erstaunlich reichhaltigen Überlegun-

\footnotetext{
${ }^{27}$ Die hier einschlägige Literatur ist längst zu umfangreich, als dass man sie an dieser Stelle referieren könnte. Sie kommt übrigens von „links" wie von "rechts“ und enthält auch Überlegungen dazu, wie man die Kosten eines Ausscheidens aus der Währungsunion für das betreffende Land mindestens teilweise denjenigen Ländern aufbürden könnte, von deren unrealistischen expliziten und impliziten Versprechungen die Weichwährungsländer überhaupt erst in die Währungsunion gelockt wurden. Siehe insbesondere Flassbeck und Lapavitsas (2015, mit einem Vorwort von Oskar Lafontaine) sowie laufende Diskussionsbeiträge wie die des amerikanischen Ökonomen Allan Meltzer (Frankfurter Allgemeine Sonntagszeitung, 16. 11. 2014), der niederländischen Ökonomen und Publizisten um André van Dam („The Matheo Solution“) sowie, unter vielen anderen, Wolfgang Münchau (Financial Times, 16. März 2015).
} 
gen der Zwischenkriegszeit über alternative nationale und supranationale Geldregime anknüpfen (z. B. Fisher 1936; Keynes 1930). Aus ihnen ließe sich mindestens lernen, dass Geld eine historisch gewachsene, sich ständig entwickelnde und immer wieder gestaltungsbedürftige Institution ist, die nicht nur effizienztheoretisch, sondern auch in Bezug auf ihre politischen Funktionen beurteilt werden kann und muss. Die Zukunft der Europäischen Währungsunion könnte so zum Unterthema einer weltweiten Debatte über eine Geld- und Kreditordnung für den Kapitalismus, und vielleicht sogar den Post-Kapitalismus, des 21. Jahrhunderts werden. ${ }^{28}$

Oder auch nicht. Nach wie vor und mehr denn je gilt, dass zwischen den sich verschärfenden Reproduktionsproblemen des Kapitalismus der Gegenwart und der für ihre Lösung verfügbaren kollektiven Handlungsfähigkeit ein groteskes Missverhältnis besteht - und nicht nur, was die Reparatur des Geldes betrifft, sondern auch in Bezug auf die Regulierung der Ausbeutung von Arbeitskraft und Natur (Streeck 2014). Für dürfte dies bedeuten, dass wir jedenfalls für die nächsten zwei bis drei Jahrzehnte nicht damit rechnen können, dass diejenigen, die uns den Euro beschert haben, auch nur ernsthaft versuchen, geschweige denn in der Lage sein werden, uns vor ihm in Sicherheit zu bringen. Die Zauberlehrlinge werden den Besen, mit dem sie Europa um der neoliberalen Transformation seines Kapitalismus willen von seinen vormodernen sozialen und antikapitalistischen Mucken säubern wollten, nicht mehr loswerden. So wird sich die politische Spaltungsmaschine des Euro weiterhin ungehindert in unseren Kontinent fressen können. Das plausibelste Szenario für das Europa der nahen und nicht-so-nahen Zukunft ist eines wachsender wirtschaftlicher Disparitäten und zunehmender politischer und kultureller Feindseligkeit zwischen den europäischen Völkern,

\footnotetext{
${ }^{28}$ Tagesordnungspunkte wären unter anderem die Ablösung des Dollars als Reservewährung, die Ermächtigung von Staaten und internationalen Organisationen zur Begrenzung der Kapitalverkehrsfreiheit, die Regulierung des Schattenbankenunwesens und der globalen Geld- und Kreditschöpfung sowie der Festsetzung der Wechselkurse.
} 
flankiert von technokratischen Entdemokratisierungsversuchen einerseits und dem Aufstieg neuer nationalistischer Massenparteien andererseits, die die Gelegenheit nutzen werden, sich zu authentischen Repräsentanten der von einer markt- und globalisierungsfreundlich gewendeten Linken verlassenen wachsenden Zahl sogenannter Modernisierungsverlierer zu erklären. Besonders ungemütlich wird diese Welt, die dazu noch unter der Dauerdrohung jederzeit möglicher Wiederholungen von 2008 stünde, für die Deutschen, die um des Euro willen ohne jenes „Europa“ werden auskommen müssen, von dem sie sich einmal ihre sichere Einhausung im Kreis freundlich gesinnter Nachbarn versprochen hatten.

\section{Literatur}

Armingeon, Klaus und Lucio Baccaro, 2012: Political Economy of the Sovereign Debt Crisis: The Limits of Internal Devaluation. Industrial Law Journal. Jg. 41, Nr. 3, 254-275.

Baccaro, Lucio und Chiara Benassi, 2014: Softening Industrial Relations Institutions, Hardening Growth Model: The Transformation of the German Political Economy. Stato e mercato. Nr. 102, 369-395.

Blankart, Charles B., 2013 Oil and Vinegar: A Positive Fiscal Theory of the Euro Crisis. Kyklos. Jg. 66, Nr. 3, 497-528.

Carruthers, Bruce G. und Sarah Babb, 1996: The Color of Money and the Nature of Value: Greenbacks and Gold in Postbellum America. American Journal of Sociology. Jg. 101, Nr. 6, 1556-1591.

Dodd, Nigel, 1994: The Sociology of Money: Economics, Reason \& Contemporary Society. New York: Continuum.

Fisher, Irving, 1936: 100\% Money. New York: Adelphi Company.

Flassbeck, Heiner und Costas Lapavitsas, 2015: Nur Deutschland kann den Euro retten: Der letzte Akt beginnt. Frankfurt am Main: Westend.

Habermas, Jürgen, 2014: Warum der Ausbau der Europäischen Union zu einer supranationalen Demokratie nötig und wie er möglich ist. Leviathan. Jg. 42, Nr. 4, 524-538. 
Habermas, Jürgen, 2014 [1987]: Theorie des kommunikativen Handelns. Band 2: Zur Kritik der funktionalistischen Vernunft. Berlin: suhrkamp taschenbuch wissenschaft 1175.

Hacker, Jacob und Paul Pierson, 2014: After the "Master Theory": Downs, Schattschneider, and the Rebirth of Policy-Focused Analysis. Perspectives on Politics. Jg. 12 Nr. 3, 643-662.

Hall, Peter, 2012 The Economics and Politics of the Euro Crisis. German Politics. Jg. 21, Nr. 4, 355-371.

Hancké, Bob, 2013: Unions, Central Banks, and EMU. Labor Market Institutions and Monetary Integration in Europe. Oxford: Oxford University Press.

Hank, Rainer, 2015: Europa der Heuchler. Frankfurter Allgemeine Zeitung, 15. März 2015,

Hirschman, Albert O., 1982: Rival Interpretations of Market Society: Civilizing, Destructive, or Feeble? Journal of Economic Literature. Jg. 20, Nr. 4, 1463-1484

Höpner, Martin und Mark Lutter, 2014: One Currency and Many Modes of Wage Formation. Why the Eurozone is too Heterogeneous for the Euro. MPIfG Discussion Paper Nr. 14/14. Max-PlanckInstitut für Gesellschaftsforschung Köln.

Ingham, Geoffrey, 2004: The Nature of Money. Cambridge: Polity.

Johnston, Alison und Aidan Regan, 2014: European integration and the incompatibility of different varieties of capitalism: Problems with institutional divergence in a monetary union. MPIfG Discussion Paper Nr. 14/15. Max-Planck-Institut für Gesellschaftsforschung Köln.

Keynes, John Maynard, 1930: A Treatise on Money. New York: Harcourt, Brace and Company.

Knapp, Georg Friedrich, 1905: Staatliche Theorie des Geldes. München und Leipzig: Duncker \& Humblot.

Knight, Jack, 1992: Institutions and Social Conflict. Cambridge: Cambridge University Press.

Mertens, Daniel, 2014: Privatverschuldung in Deutschland: Institutionalistische und vergleichende Perspektiven auf die Finanzialisierung privater Haushalte. Doctoral Dissertation. Köln: MaxPlanck-Institut für Gesellschaftsforschung und Wirtschafts- und sozialwissenschaftliche Fakultät der Universität Köln.

Parsons, Talcott, 1964: Evolutionary Universals in Society. American Sociological Review. Jg. 29, Nr. 3, 339-357. 
Parsons, Talcott und Neil J. Smelser, 1984 [1956]: Economy and Society: A Study in the Integration of Economic and Social Theory. London etc.: Routledge and Kegan Paul.

Polanyi, Karl, 1957 [1944]: The Great Transformation: The Political and Economic Origins of Our Time. Boston: Beacon Press.

Scharpf, Fritz W., 2013: Political Legitimacy in a Non-optimal Currency Area. MPIfG Discussion Paper Nr. 13/15. Max-Planck-Institut für Gesellschaftsforschung Köln.

Scharpf, Fritz W., 2015: Das Dilemma der supranationalen Demokratie in Europa. Leviathan. Jg. 42, Nr. im Erscheinen.

Schattschneider, E. E., 1960: The Semi-Sovereign People. New York: Holt, Reinhart, and Winston.

Smith, Adam, 1993 [1776]: An Inquiry into the Nature and Causes of the Wealth of Nations. Oxford and New York: Oxford University Press.

Streeck, Wolfgang, 2011: E Pluribus Unum? Varieties and Commonalities of Capitalism. In: Granovetter, Mark und Richard Swedberg (Hg.), The Sociology of Economic Life. 3rd edition. Boulder, Colorado: Westview, 419-455.

Streeck, Wolfgang, 2013: Gekaufte Zeit: Die vertagte Krise des demokratischen Kapitalismus. Berlin: Suhrkamp.

Streeck, Wolfgang, 2014: How Will Capitalism End? New Left Review. Jg. 87, Nr. May/June 2014, $35-$ 64.

Streeck, Wolfgang, 2015a: Comment on Wolfgang Merkel, "Is Capitalism Compatible with Democracy?". Zeitschrift für vergleichende Politikwissenschaft. Published online, 7 February, 2015.

Streeck, Wolfgang, 2015b: The Rise of the European Consolidation State. In: King, Desmond und Patrick Le Galés (Hg.), The Reconfiguration of the State in Europe. Oxford: Oxford University Press.

Streeck, Wolfgang und Lea Elsässer, 2014: Monetary Disunion: The Domestic Politics of Euroland. MPIfG Discussion Paper Nr. 14-17. Max-Planck-Institut für Gesellschaftsforschung Köln.

Weber, Max, 1956 [1920]: Wirtschaft und Gesellschaft. Erster Halbband. Köln: Kiepenheuer \& Witsch. 